\title{
Millimeter and Submillimeter Observations from the South Pole
}

\author{
Antony A. Stark \\ Smithsonian Astrophysical Observatory, 60 Garden St., Cambridge, MA 02138 USA
}

\begin{abstract}
During the past decade, a year-round observatory has been established at the geographic South Pole by the Center for Astrophysical Research in Antarctica (CARA). CARA has fielded several millimeter- and submillimeter-wave instruments: AST/RO (the Antarctic Submillimeter Telescope and Remote Observatory, a $1.7-\mathrm{m}$ telescope outfitted with a variety of receivers at frequencies from $230 \mathrm{GHz}$ to $810 \mathrm{GHz}$, including PoleSTAR, a heterodyne spectrometer array), Python (a degree-scale CMB telescope), Viper (a 2-m telescope which has been outfitted with SPARO, a submillimeter-wave bolometric array polarimeter, ACBAR, a multichannel CMB instrument, and Dos Equis, a HEMT polarimeter), and DASI (the Degree-Angular Scale Interferometer). These instruments have obtained significant results in studies of the interstellar medium and observational cosmology, including detections of the $1^{\circ}$ acoustic peak in the CMB and the Sunyaev-Zel'dovich effect. The South Pole environment is unique among observatory sites for unusually low wind speeds, low absolute humidity, and the consistent clarity of the submillimeter sky. The atmosphere is dessicated by cold: at the South Pole's average annual temperature of $-49 \mathrm{C}$, the partial pressure of saturated water vapor is only $1.2 \%$ of what it is at $0 \mathrm{C}$. The low water vapor levels result in exceptionally low values of sky noise. This is crucial for large-scale observations of faint cosmological sources-for such observations the South Pole is unsurpassed.
\end{abstract}

\section{INTRODUCTION}

Development of the geographic South Pole as an astronomical observatory has largely been driven by the desire to measure anisotropy in the Cosmic Microwave Background (CMB) radiation. Observers have actively pursued the measurement of CMB anisotropy because of the theoretical expectation that those measurements would significantly advance our understanding of the Universe [1]. Deep millimeter- and submillimeter-wave observations, of which CMB anisotropy measurements are an extreme example, are often made impossible by opacity and noise due to water vapor in the Earth's atmosphere [2]. For large angular scale measurements, this problem has been successfully finessed by orbital [3] and airborne [4,5] instruments, but observations of anisotropy on scales less than a few arcminutes require large telescope apertures, which cannot be deployed above the Earth's surface using current technology. This hard truth has led observers to seek new observatory sites having the smallest possible atmospheric water vapor while still being practical locations for the installation and operation of large telescopes. Among the most promising of the new observatories is Amundsen-Scott South Pole Station, the permanent U.S. National Science Foundation base on the Antarctic Plateau.

South Pole Station was originally built for the International Geophysical Year in 1957 and has been in continuous use ever since. Station infrastructure is currently being rebuilt for the second time; the new station is scheduled for completion in 2005. The station supports 50 scientists and staff throughout the Austral winter, with an increase to over 200 during the summer months. From the earliest days, scientific investigations at South Pole Station included observations of weather [6,7] and atmospheric phenomena such as aurora [8] and ice halos [9]. The South Pole has evolved into an important site for seismology [10], solar astronomy [11, 12], atmospheric studies [13], and cosmic ray physics [14].

Because it is exceptionally cold, the climate at the Pole implies exceptionally dry observing conditions. As air becomes cold, the amount of water vapor it can hold declines dramatically. Air at $0 \mathrm{C}$ can hold 83 times more water vapor than saturated air at the South Pole's average annual temperature of -49 C [15]; together with the relatively high altitude of the Pole $(2850 \mathrm{~m})$, this means the water vapor content of the atmosphere above the South Pole is two 
or three orders of magnitude smaller than it is at most places on the Earth's surface. This has long been known [16], but many years of hard work were needed to realize the potential in the form of new astronomical knowledge.

The EMILIE experiment by the French group Pajot et al. [17] made the first astronomical observations of submillimeter-waves from the South Pole during the Austral summer of 1984-1985. EMILIE was a ground-based single-pixel bolometer dewar operating at $900 \mu \mathrm{m}$ and fed by a $45 \mathrm{~cm}$ off-axis mirror. It had successfully measured the diffuse galactic emission while operating on Mauna Kea in Hawaii in 1982, but the accuracy of the result had been limited by sky noise [18]. Martin A. Pomerantz, a cosmic ray researcher at Bartol Research Institute, and John T. Lynch, the NSF Program Director for Antarctic Aeronomy and Astrophysics [19], encouraged the EMILIE group to relocate their experiment to the South Pole. There they found better observing conditions and were able to make improved measurements of galactic emission [20].

Pomerantz also enabled Mark Dragovan, then a researcher at Bell Laboratories, to attempt CMB anisotropy measurements from the Pole. Dragovan, Stark, and Pernic [21] built a lightweight $1.2 \mathrm{~m}$ offset telescope and were able to get it working at the Pole with a single-pixel helium-4 bolometer during several weeks in January 1987. The results were sufficiently encouraging that several CMB groups [22, 23, 24, 25] participated in the "Cucumber" campaign in the Austral summer of 1988-1989, where three Jamesway tents and a generator dedicated to CMB anisotropy measurements were set up at a temporary site $2 \mathrm{~km}$ from South Pole Station. These were summer-only campaigns, where instruments were shipped in, assembled, tested, used, disassembled, and shipped out in a single three-monthlong summer season. Considerable time and effort were expended in establishing and then demolishing observatory facilities, with little return in observing time. What little observing time was available occurred during the warmest and wettest days of mid-summer.

Permanent, year-round facilities were needed. The Antarctic Submillimeter Telescope and Remote Observatory (AST/RO) $[26,27]$ is a $1.7 \mathrm{~m}$ diameter offset gregorian telescope mounted on a dedicated, permanent observatory building. It was the first radio telescope to operate year-round at South Pole. AST/RO was started in 1989 as an independent project, but in 1991 it became part of a newly-founded National Science Foundation Science and Technology Center, the Center for Astrophysical Research in Antarctica (CARA) [28, see also http: //astro. uchicago.edu/cara]. CARA has fielded several telescopes in addition to AST/RO: White Dish [29], Python [30, 31, 32, 33, 34] and Viper [35] (Cosmic Microwave Background experiments), DASI [36] (the Degree-Angular Scale Interferometer), and SPIREX [37] (the South Pole Infrared Explorer, a 60-cm telescope, now decommissioned). These facilities are housed in the "Dark Sector", a grouping of buildings which includes the AST/RO building, the Martin A. Pomerantz Observatory building (MAPO) and a new "Dark Sector Laboratory", all located $1 \mathrm{~km}$ away from the main base across the aircraft runway in a radio quiet zone.

\section{SITE TESTING}

The South Pole is an excellent millimeter- and submillimeter-wave site [39]. It is unique among observatory sites for unusually low wind speeds, the complete absence of rain, and the consistent clarity of the submillimeter sky. Schwerdtfeger [6] has comprehensively reviewed the climate of the Antarctic Plateau and the records of the South Pole meteorology office. Chamberlin [40] has analyzed weather data to determine the precipitable water vapor (PWV) and finds median wintertime PWV values of $0.3 \mathrm{~mm}$ over a 37 -year period, with little annual variation. $P W V$ values at South Pole are small, stable, and well-understood.

Submillimeter-wave atmospheric opacity at South Pole has been measured using skydip techniques. Chamberlin et al. [41] made over 1100 skydip observations at $492 \mathrm{GHz}(609 \mu \mathrm{m})$ with AST/RO during the 1995 observing season. Even though this frequency is near a strong oxygen line, the opacity was below 0.70 half of the time during the Austral winter and reached values as low as 0.34 , better than ever measured at any other ground-based site. From early 1998, the $350 \mu \mathrm{m}$ band has been continuously monitored at Mauna Kea, Chajnantor, and South Pole by identical tipper instruments developed by S. Radford of NRAO and J. Peterson of Carnegie-Mellon U. and CARA. Results from Mauna Kea and Chajnantor are compared with South Pole in the left panel of Figure 1. The $350 \mu \mathrm{m}$ opacity at the South Pole is consistently better than at Mauna Kea or Chajnantor. The South Pole 25\% winter PWV levels have been used to compute values of atmospheric transmittance as a function of wavelength and are plotted in the right panel of Figure 1. For comparison, the transmittance for $25 \%$ winter conditions at Chajnantor and Mauna Kea is also shown.

Sky noise is fluctuations in total power or phase of a detector caused by variations in atmospheric emissivity and path length on timescales of order one second. Sky noise causes systematic errors in the measurement of astronomical sources. Lay and Halverson [42] show analytically how sky noise causes observational techniques to fail: fluctuations 

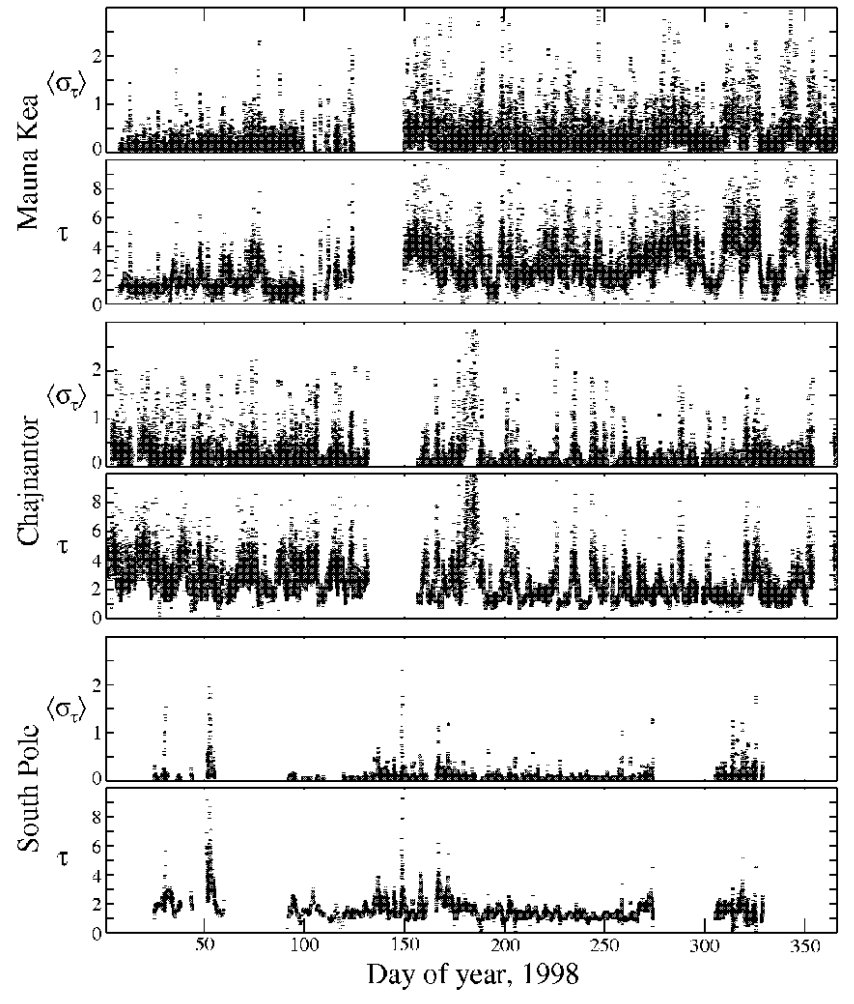

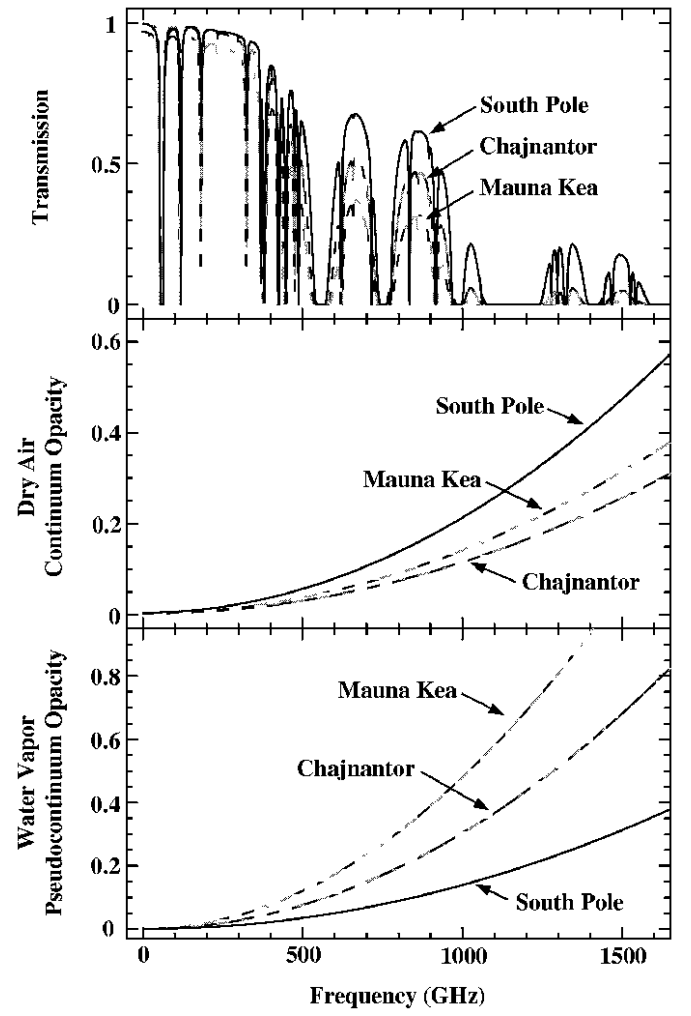

FIGURE 1. Opacity and sky noise at the South Pole. (Left) Measurements at $350 \mu \mathrm{m}$ from three sites. These plots show data from identical NRAO-CMU 350 $\mu \mathrm{m}$ broadband tippers located at Mauna Kea, the ALMA site at Chajnantor in Chile, and South Pole during 1998. The upper plot of each pair shows $\left\langle\sigma_{\tau}\right\rangle$, the rms deviation in the opacity $\tau$ during a one-hour period-a measure of sky noise on large scales; the lower plot of each pair shows $\tau$, the broadband 350 $\mu \mathrm{m}$ opacity. The first 100 days of 1998 on Mauna Kea were exceptionally good for that site, due to a strong El Niño that year. During the best weather at the Pole, $\left\langle\sigma_{\tau}\right\rangle$ was dominated by detector noise rather than sky noise. (Right) Calculated atmospheric transmittance at the three sites. The upper plot is atmospheric transmittance at zenith calculated by J. R. Pardo using the ATM model [38]. The model uses PWV values of $0.2 \mathrm{~mm}$ for South Pole, $0.6 \mathrm{~mm}$ for Chajnantor and $0.9 \mathrm{~mm}$ for Mauna Kea, corresponding to the $25^{\text {th }}$ percentile winter values at each site. Note that at low frequencies, the Chajnantor curve converges with the South Pole curve, an indication that $225 \mathrm{GHz}$ opacity is not a simple predictor of submillimeter wave opacity. The middle and lower plots show calculated values of dry air continuum opacity and water vapor pseudocontinuum opacity for the three sites. Note that unlike the other sites, the opacity at South Pole is dominated by dry air rather than water vapor.

in a component of the data due to sky noise integrates down more slowly than $t^{-1 / 2}$ and will come to dominate the error during long observations. Sky noise at South Pole is considerably smaller than at other sites, even comparing conditions of the same opacity. The PWV at South Pole is often so low that the opacity is dominated by the dry air component [43, 40, cf. Figure 1]; the dry air emissivity and phase error do not vary as strongly or rapidly as the emissivity and phase error due to water vapor. Lay and Halverson [42] have compared the Python experiment at South Pole with the Site Testing Interferometer at Chajnantor $[44,45]$ and find that the amplitude of the sky noise at South Pole is 10 to 50 times less than that at Chajnantor. The strength of South Pole as a millimeter and submillimeter site lies in the low sky noise levels routinely obtainable for sources around the South Celestial Pole.

\section{LOGISTICS}

South Pole Station provides logistical support for observatory experiments: room and board for on-site scientific staff, electrical power, network and telephone connections, heavy equipment support, and cargo and personnel transport. The station powerplant provides about $100 \mathrm{~kW}$ of power to CARA projects out of a total generating capacity of about $600 \mathrm{~kW}$. Heavy equipment at South Pole Station includes cranes, forklifts, and bulldozers; these can be requisitioned 


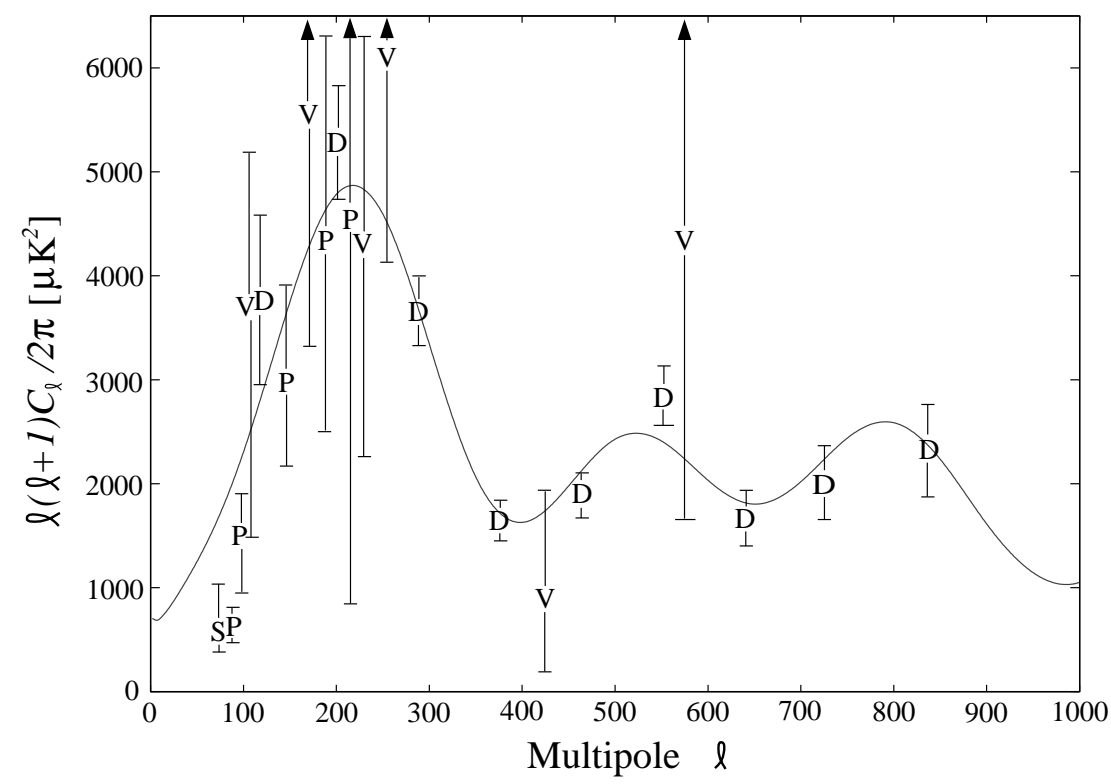

FIGURE 2. Summary of Cosmic Microwave Background Anisotropy measurements from the South Pole. The data shown are: $\mathrm{S}=\mathrm{U}$. of California, Santa Barbara [46]; P = Python 1991-1996 [34]; V = Viper 1998 [35]; D = DASI 2000 [47]. The curved line is not a fit to the data, but instead shows the expected values for the cosmological "concordance model": $\Omega_{\mathrm{b}}=0.05, \Omega_{\mathrm{cdm}}=0.35$, $\Omega_{\Lambda}=0.60, \tau_{\mathrm{c}}=0, n_{\mathrm{s}}=1.00, H_{0}=65 \mathrm{~km} \mathrm{~s}^{-1} \mathrm{kpc}^{-1}$ [48]. The data are in remarkably good agreement with the concordance model, and strongly reject many other cosmologies.

for scientific use as needed. The station is supplied by over 200 flights each year of LC130 ski-equipped cargo aircraft. Annual cargo capacity is about 3500 tons. Aircraft flights are scheduled only during the period from late October to early February so that the station is inaccessible for as long as nine months of the year. All engineering operations for equipment installation and maintenance are tied to the annual cycle of physical access to the instruments. For quick repairs and upgrades during the Austral summer season, it is possible to send equipment between South Pole and anywhere serviced by commercial express delivery in about five days. During the winter, however, no transport is possible and projects must be designed and managed accordingly.

In summer, there are about 20 CARA people at the Pole at any given time. Each person stays at Pole for a few weeks or months in order to carry out their planned tasks as well as circumstances allow, then they depart to be replaced by another CARA person. Each year there are four or five CARA winter-over scientists, who remain at the observatory for a year.

The receivers used on AST/RO and Viper require about 20 liters of liquid helium per day. Helium also escapes from the station in one or two weather balloons launched each day. The National Science Foundation and its support contractors must supply helium to South Pole, and the most efficient way to transport and supply helium is in liquid form. Before the winter-over period, one or more large (4000 to 12000 liter) storage dewars are brought to South Pole for winter use; some years this supply lasts the entire winter, but in 1996, 2000, and 2001 it did not. The supply of liquid helium has been a chronic problem for South Pole astronomy, but improved facilities in the new station should eliminate single points of failure and provide a more certain supply.

Internet and telephone service to South Pole is provided by a combination of two low-bandwidth satellites, LES-9 and GOES-3, and the high-bandwidth (3 Mbps) NASA Tracking and Data Relay Satellite System TDRS-F1. These satellites are geosynchronous but not geostationary, since their orbits are inclined. Geostationary satellites are always below the horizon and cannot be used. Internet service is intermittent through each 24-hour period because each satellite is visible only during the southernmost part of its orbit; the combination of the three satellites provides an Internet connection for approximately 12 hours within the period 1-16 hr Greenwich LST. The TDRS link helps provide a store-and-forward automatic transfer service for large computer files. The total data communications capability is about 5 Gbytes per day. Additional voice communications are provided by a fourth satellite, ATS-3, and high frequency radio. 


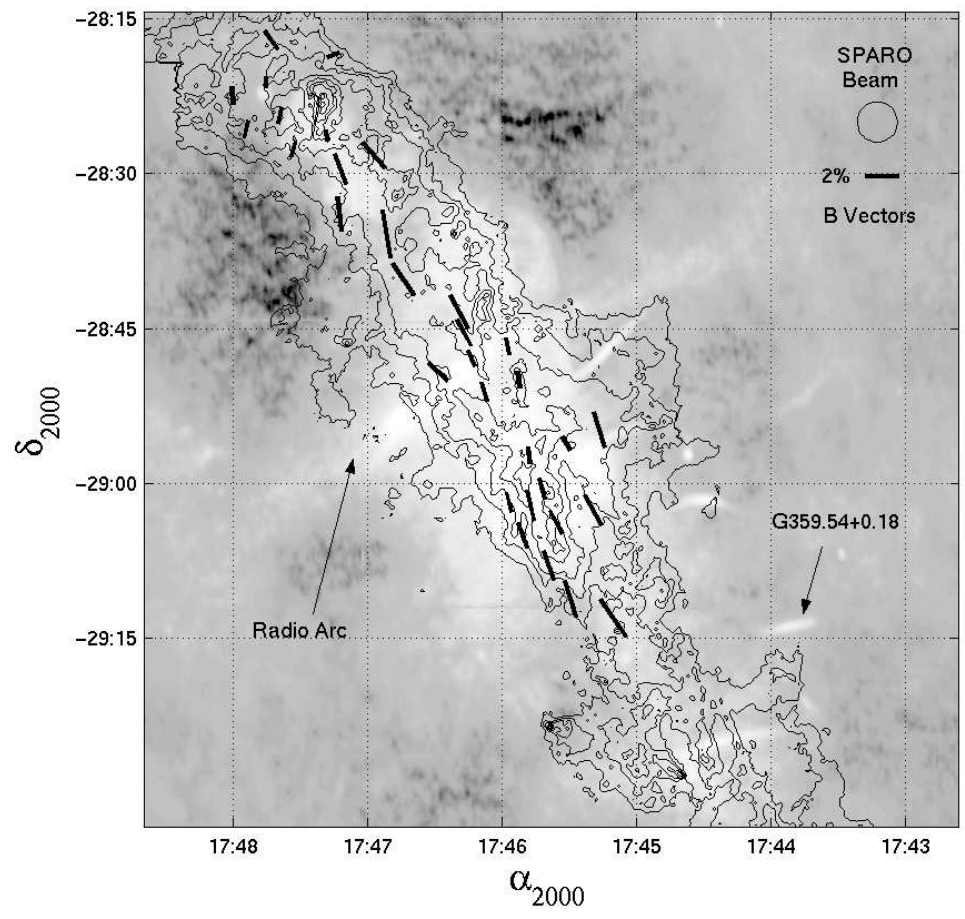

Source: G. Novak

FIGURE 3. SPARO polarimetry results from Novak et al. [49] for the Galactic Center Region (vectors) superposed on radio continuum map by LaRosa et al. [50] (gray scale). The length of each vector gives the measured degree of polarization (see key at upper right), and the orientation of each vector shows the direction of the inferred magnetic field, which is orthogonal to the measured direction of polarization. Black contours show the $800 \mu \mathrm{m}$ dust emission measured using the JCMT [51]. The radio continuum map shows several of the non-thermal filaments that trace magnetic field lines in hot ionized regions.

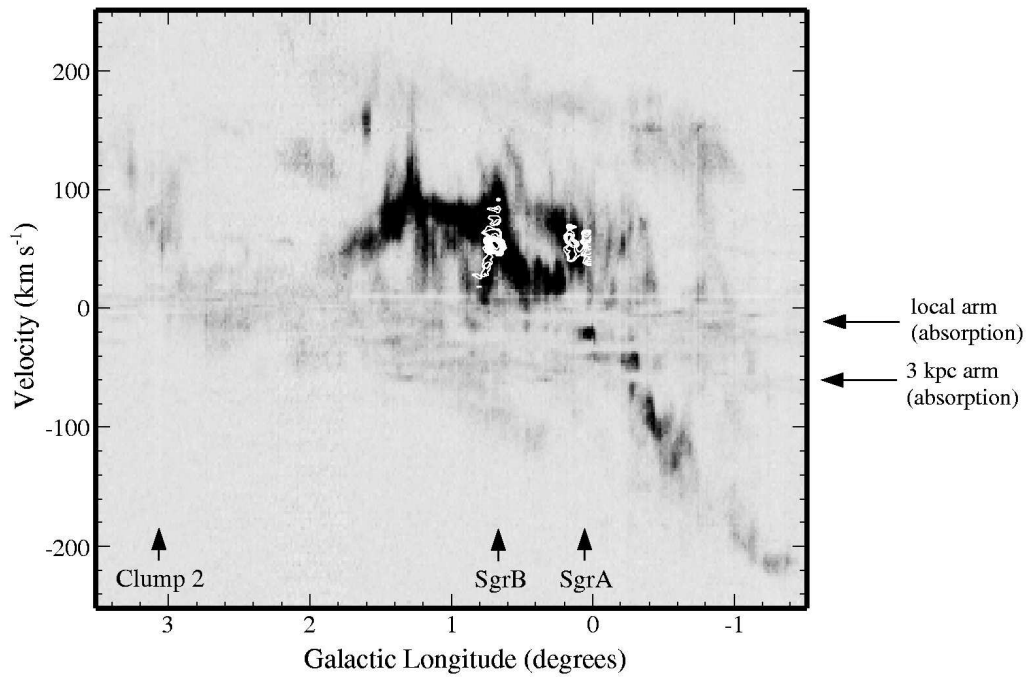

Source: S. Kim

FIGURE 4. AST/RO observations of the Galactic Center Region from Kim et al. [52]. The CO $J=4 \rightarrow 3$ (greyscale) and $J=7 \rightarrow 6$ (white contour) lines observed in an $l-v$ strip, sampled every $1^{\prime}$, at $b=0^{\circ}$. These data have been used in conjunction with $\mathrm{CO}$ and ${ }^{13} \mathrm{CO} J=1 \rightarrow 0$ data to determine the the density and temperature in the features shown here [52]. The 300 parsec ring is the rough parallelogram-shape between $l=-1^{\circ}$ and $l=1.8^{\circ}$, and velocities from -200 to $+200 \mathrm{~km} \mathrm{~s}^{-1}$. The ring is just below the critical density at which instabilities will cause it to coagulate into a single cloud like Sgr B and Sgr A. 


\section{CURRENT INSTRUMENTS AND ILLUSTRATIVE RESULTS}

The Degree Angular Scale Interferometer (DASI) [36] is a compact centimeter-wave interferometer designed to image the CMB primary anisotropy and measure its angular power spectrum and polarization at angular scales ranging from two degrees to several arcminutes. As an interferometer, DASI measures CMB power by simultaneous differencing on several scales, measuring the CMB power spectrum directly. DASI was installed on a tower adjacent to the MAPO during the 1999-2000 Austral summer and has had two successful winter seasons so far. The first season results are a stunning confirmation of the "concordance" cosmological model, which has a flat geometry and significant contributions to the total stress-energy from dark matter and dark energy [47, 48].

Viper is a 2.1 meter off-axis telescope designed to allow measurements of low contrast astronomical sources. It is mounted on another tower at the opposite end of the MAPO from DASI. Viper is used with a variety of instruments: Dos Equis, a CMB polarization receiver operating at $7 \mathrm{~mm}$, SPARO, a bolometric array polarimeter operating at 450 $\mu \mathrm{m}$, and ACBAR, a multi-wavelength bolometer array used to map the CMB.

ACBAR is a 16-element bolometer array operating at $300 \mathrm{mK}$. It was designed specifically for observations of CMB anisotropy and the Sunyaev-Zel'dovich effect (SZE). It was installed on the Viper telescope during the Austral summer of 2000-2001 and was successfully operated throughout most of the Austral winter of 2001. ACBAR has made highquality maps of SZE in several nearby clusters of galaxies and has made significant measurements of anisotropy on the scale of degrees to arcminutes.

The Submillimeter Polarimeter for Antarctic Remote Observing (SPARO) is a 9-pixel polarimetric imager operating at $450 \mu \mathrm{m}$. It was operational on the Viper telescope during the early Austral winter of 2000. Novak et al. [49] mapped the polarization of an extended region of the sky $(\sim 0.25$ square degrees $)$ centered approximately on the Galactic Center. Their results imply that, within the Galactic Center molecular gas complex, the toroidal component of the magnetic field is dominant. The data show that all of the existing observations of large-scale magnetic fields in the Galactic Center are basically consistent with the "magnetic outflow" model of Uchida et al. [53]. This magnetodynamic model was developed in order to explain the Galactic Center Radio Lobe, a limb-brightened radio structure that extends up to one degree above the plane and may represent a gas outflow from the Galactic Center.

AST/RO is general-purpose $1.7 \mathrm{~m}$ diameter telescope $[26,27]$ for astronomy and aeronomy studies at wavelengths between 200 and $2000 \mu \mathrm{m}$. It is used primarily for spectroscopic studies of neutral atomic carbon and carbon monoxide in the interstellar medium of the Milky Way and the Magellanic Clouds (see reference list at http:// cfa-www.harvard.edu/ adair/AST_RO). Five heterodyne receivers and three acousto-optical spectrometers are currently installed: (1) a $230 \mathrm{GHz}$ SIS receiver [54], (2) a 450-495 GHz SIS quasi-optical receiver [55, 56], (3) a 450-495 GHz SIS waveguide receiver [57, 58], which can be used simultaneously with (4) a 800-820 GHz fixed-tuned SIS waveguide mixer receiver [59], and (5) the PoleSTAR array-four 800-820 GHz fixed-tuned SIS waveguide mixer receivers [60, 61, see http: // soral. as .arizona.edu/pole-star]. Spectral lines observed with AST/RO include: $\mathrm{CO} J=7 \rightarrow 6, \mathrm{CO} J=4 \rightarrow 3, \mathrm{CO} J=2 \rightarrow 1$, HDO $J=1_{0,1} \rightarrow 0_{0,0}$, [C I ] ${ }^{3} P_{1} \rightarrow{ }^{3} P_{0}$, [C I ] ${ }^{3} P_{2} \rightarrow{ }^{3} P_{1}$, and $\left[{ }^{13} \mathrm{C} \mathrm{I}\right]{ }^{3} P_{2} \rightarrow{ }^{3} P_{1}$. There are four currently available acousto-optical spectrometers (AOS) [62]: two low resolution spectrometers with a bandwidth of $1 \mathrm{GHz}$, an array AOS having four low resolution spectrometer channels with a bandwidth of $1 \mathrm{GHz}$ for the PoleSTAR array, and one high-resolution AOS with $60 \mathrm{MHz}$ bandwidth. Two new instruments for AST/RO are under development: TREND, a $1.5 \mathrm{THz}$ heterodyne receiver $[63,64]$ and SPIFI, an imaging Fabry-Perot interferometer [65]. AST/RO observing time is open to all astronomers on a proposal basis.

\section{THE FUTURE 8-M TELESCOPE}

An $8 \mathrm{~m}$ diameter off-axis telescope has been proposed for the South Pole [66]. It will be equipped with a large field of view [67] that will feed a state-of-the-art 1027-element bolometer array receiver. The initial science goal will be a large SZE survey covering 4000 square degrees at $1.3^{\prime}$ resolution with $10 \mu \mathrm{K}$ sensitivity at a wavelength of $2 \mathrm{~mm}$. The SZE survey will find all galaxy clusters above a mass limit of $3.5 \times 10^{14} \mathrm{M}_{\odot}$ regardless of redshift; should clusters exist at redshifts much higher than currently predicted, they will be found by the SZE survey, but missed in even the deepest X-ray observations planned. It is expected that an unbiased sample of approximately 20,000 clusters will be found, with over 1,000 at redshifts greater than one. The sample will provide sufficient statistics to use the density of clusters to determine the equation of state of the dark energy component of the Universe. 


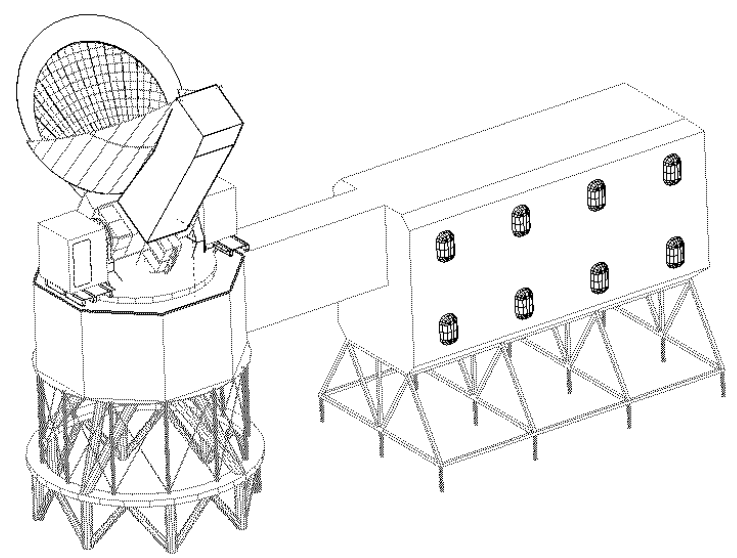

FIGURE 5. A sketch of the proposed 8-m South Pole Telescope and tower mounted to the side of the new Dark Sector Laboratory. A guard ring surrounding the primary and shielding around the lower part of the secondary arm support region minimize contamination from scattered radiation. The guard ring allows the full aperture to be illuminated without excessive noise from spillover. Like the DASI and AST/RO telescopes, the environment of all critical and moving components will be kept warm, and therefore access will not require the winterover staff to work in the extreme cold conditions.

\section{ACKNOWLEDGMENTS}

We thank Jeff Peterson of CMU and Simon Radford of NRAO for the data shown in the left panel of Figure 1. We thank Juan R. Pardo of Caltech for discussions on atmospheric modeling and for carrying out the calculations shown in the right panel of Figure 1. This work was supported in part by the Center for Astrophysical Research in Antarctica and the NSF under Cooperative Agreement OPP89-20223.

\section{REFERENCES}

1. Peebles, P. J. E., Physical Cosmology, Princeton University Press, 1971.

2. Radford, S. J. E., Boynton, P. E., Ulich, B. L., Partridge, R. B., Schommer, R. A., Stark, A. A., Wilson, R. W., and Murray, S. S., Astrophys. J., 300, 159 (1986).

3. Smoot, G. F., et al., Astrophys. J., 396, L1 (1992).

4. de Bernardis, P., Ade, P. A. R., Bock, J. J., Bond, J. R., Borrill, J., Boscaleri, A., Coble, K., Crill, B. P., De Gasperis, G., Farese, P. C., Ferreira, P. G., Ganga, K., Giacometti, M., Hivon, E., Hristov, V. V., Iacoangeli, A., Jaffe, A. H., Lange, A. E., Martinis, L., Masi, S., Mason, P. V., Mauskopf, P. D., Melchiorri, A., Miglio, L., Montroy, T., Netterfield, C. B., Pascale, E., Piacentini, F., Pogosyan, D., Prunet, S., Rao, S., Romeo, G., Ruhl, J. E., Scaramuzzi, F., Sforna, D., and Vittorio, N., Nature, 404, 955 (2000).

5. Hanany, S., Ade, P., Balbi, A., Bock, J., Borrill, J., Boscaleri, A., de Bernardis, P., Ferreira, P. G., Hristov, V. V., Jaffe, A. H., Lange, A. E., Lee, A. T., Mauskopf, P. D., Netterfield, C. B., Oh, S., Pascale, E., Rabii, B., Richards, P. L., Smoot, G. F., Stompor, R., Winant, C. D., and Wu, J. H. P., Astrophys. J. Lett. , 545, L5 (2000).

6. Schwerdtfeger, W., Weather and Climate of the Antarctic, Elsevier, Amsterdam, 1984.

7. Warren, S. G., "Antarctica", in Encyclopedia of Weather and Climate, edited by S. H. Schneider, Oxford Univ. Press., New York, 1996, vol. 1, p. 32.

8. Landolt, A. U., Proc. Ast. Soc. Pac. , 70, 581 (1958).

9. Ohtake, T., Antarctic. J. US, 13, 174 (1978).

10. Roult, G., and Rouland, D., Physics of the Earth and Planetary Interiors, 84, 15 (1994).

11. Harvey, J., "Solar Observing Conditions at the South Pole", in Astrophysics in Antarctica, edited by D. J. Mullan, M. A. Pomerantz, and T. Stanev, AIP Press, New York, 1989, p. 227.

12. Libbrecht, K. G., and Woodard, M. F., Science, 5016, 152 (1991).

13. Fan, S. M., Blaine, T. L., and Sarmiento, J. L., Tellus Series B-Chemical and Physical Meteorology, 51, 863 (1999).

14. Smith, N. J. T., et al., Nucl. Inst. \& Meth. in Physics Res., 276, 622-7 (1989).

15. Goff, J. A., and Gratch, S., Trans. Amer. Soc. Heat. and Vent. Eng., 52, 95 (1946).

16. Smythe, W. D., and Jackson, B. V., Applied Optics, 16, 2041 (1977).

17. Pajot, F., Gispert, R., Lamarre, J. M., Peyturaux, R., Pomerantz, M. A., Puget, J.-L., Serra, G., Maurel, C., Pfeiffer, R., and Renault, J. C., Astron. Astroph. , 223, 107 (1989). 
18. Pajot, F., Gispert, R., Lamarre, J. M., Peyturaux, R., Puget, J.-L., Serra, G., Coron, N., Dambier, G., Leblanc, J., Moalic, J. P., Renault, J. C., and Vitry, R., Astron. Astroph. , 154, 55 (1986).

19. Lynch, J. T., "Astronomy \& Astrophysics in the U. S. Antarctic Program", in Astrophysics from Antarctica, edited by G. Novak and R. H. Landsberg, ASP, San Francisco, 1998, vol. 141 of ASP Conf. Ser., p. 54.

20. Pajot, F., Gispert, R., Lamarre, J. M., Peyturaux, R., and Pomerantz, M. A., Astron. Astroph. , 223, $107-111$ (1989).

21. Dragovan, M., Stark, A. A., Pernic, R., and Pomerantz, M. A., Appl. Opt. , 29, 463 (1990).

22. Dragovan, M., Platt, S. R., Pernic, R. J., and Stark, A. A., "South Pole Submillimeter Isotropy Measurements of the Cosmic Microwave Background", in Astrophysics in Antarctica, edited by D. J. Mullan, M. A. Pomerantz, and T. Stanev, AIP Press, New York, 1990, p. 97.

23. Gaier, T., Schuster, J., and Lubin, P., "Cosmic Background Anisotropy Studies at $10^{\circ}$ Angular Scales with a HEMT Radiometer", in Astrophysics in Antarctica, edited by D. J. Mullan, M. A. Pomerantz, and T. Stanev, AIP Press, New York, 1989, p. 84.

24. Meinhold, P. R., Lubin, P. M., Chingcuanco, A. O., Schuster, J. A., and Seiffert, M., "South Pole Studies of the Anisotropy of the Cosmic Microwave Background at One Degree", in Astrophysics in Antarctica, edited by D. J. Mullan, M. A. Pomerantz, and T. Stanev, AIP Press, New York, 1989, p. 88.

25. Peterson, J., "Millimeter and Sub-millimeter Photometry from Antarctica", in Astrophysics in Antarctica, edited by D. J. Mullan, M. A. Pomerantz, and T. Stanev, AIP Press, New York, 1989, p. 116.

26. Stark, A. A., Chamberlin, R. A., Cheng, J., Ingalls, J., and Wright, G., Rev. Sci. Instr., 68, 2200 (1997).

27. Stark, A. A., Bally, J., Balm, S. P., Bania, T. M., Bolatto, A. D., Chamberlin, R. A., Engargiola, G., Huang, M., Ingalls, J. G., Jacobs, K., Jackson, J. M., Kooi, J. W., Lane, A. P., Lo, K.-Y., Marks, R. D., Martin, C. L., Mumma, D., Ojha, R., Schieder, R., Staguhn, J., Stutzki, J., Walker, C. K., Wilson, R. W., Wright, G. A., Zhang, X., Zimmermann, P., and Zimmermann, R., Proc. Ast. Soc. Pac. , 113, 567 (2001).

28. Novak, G., and Landsberg, R. H., editors, Astrophysics from Antarctica, ASP Conf. Ser. 141, San Francisco: ASP, 1998.

29. Tucker, G. S., Griffin, G. S., Nguyen, H. T., and Peterson, J. S., Astrophys. J. Lett. , 419, L45 (1993).

30. Dragovan, M., Ruhl, J., Novak, G., Platt, S. R., Crone, B., Pernic, R., and Peterson, J., Astrophys. J. Lett. , 427, L67 (1994).

31. Alvarez, D. L., Measurements of the Anisotropy in the Microwave Background on Multiple Angular Scales with the Python Telescope, Ph.D. thesis, Princeton University (1995).

32. Ruhl, J. E., Dragovan, M., Platt, S. R., Kovac, J., and Novak, G., Astrophys. J., 453, L1 (1995).

33. Platt, S. R., Kovac, J., Dragovan, M., Peterson, J. B., and Ruhl, J. E., Astrophys. J. Lett. , 475, L1 (1997).

34. Coble, K., Dragovan, M., Kovac, J., Halverson, N. W., Holzapfel, W. L., Knox, L., Dodelson, S., Ganga, K., Alvarez, D., Peterson, J. B., Griffin, G., Newcomb, M., Miller, K., Platt, S. R., and Novak, G., Astrophys. J. Lett. , 519, L5 (1999).

35. Peterson, J. B., Griffin, G. S., Newcomb, M. G., Alvarez, D. L., Cantalupo, C. M., Morgan, D., Miller, K. W., Ganga, K., Pernic, D., and Thoma, M., Astrophys. J. Lett. , 532 (2000).

36. Leitch, E. M., Pryke, C., Halverson, N. W., Kovac, J., Davidson, G., LaRoque, S., Schartman, E., Yamasaki, J., Carlstrom, J. E., Holzapfel, W. L., Dragovan, M., Cartwright, J. K., Mason, B. S., Padin, S., Pearson, T. J., Shepherd, M. C., and Readhead, A. C. S., Astrophys. J. (2001), in press, astro-ph/0104488.

37. Nguyen, H. T., Rauscher, B. J., Severson, S. A., Hereld, M., Harper, D. A., Lowenstein, R. F., Morozek, F., and Pernic, R. J., Proc. Ast. Soc. Pac. , 108, 718 (1996).

38. Pardo, J. R., Cernicharo, J., and Serabyn, E., IEEE Trans. Antennas and Propagation (2001), in press.

39. Lane, A. P., "Submillimeter Transmission at South Pole", in Astrophysics from Antarctica, edited by G. Novak and R. H. Landsberg, ASP, San Francisco, 1998, vol. 141 of ASP Conf. Ser., p. 289.

40. Chamberlin, R. A., "Comparisons of saturated water vapor column from radiosonde, and mm and submm radiometric opacities at the South Pole", in Characterization and Atmospheric Transparency in the $\mathrm{mm} /$ submm Range, edited by Vernin, Munoz-Tunon, and Benkhaldoun, Astr. Soc. of the Pacific, 2001, vol. IAU SITE2000 of ASP Conference Series.

41. Chamberlin, R. A., Lane, A. P., and Stark, A. A., Astrophys. J. , 476, 428 (1997).

42. Lay, O. P., and Halverson, N. W., Astrophys. J. , 543, 787 (2000).

43. Chamberlin, R. A., and Bally, J., Int. J. Infrared and Millimeter Waves, 16, 907 (1995).

44. Radford, S. J. E., Reiland, G., and Shillue, B., PASP, 108, 441 (1996).

45. Holdaway, M. A., Radford, S. J. E., Owen, F. N., and Foster, S. M., Fast switching phase calibration: Effectiveness at Mauna Kea and Chajnantor, Millimeter Array Technical Memo 139, NRAO (1995).

46. Ganga, K., Ratra, B., Gundersen, J. O., and Sugiyama, N., Astrophys. J. , 484, 7 (1997).

47. Halverson, N. W., Leitch, E. M., Pryke, C., Kovac, J., Carlstrom, J. E., Holzapfel, W. L., Dragovan, M., Cartwright, J. K., Mason, B. S., Padin, S., Pearson, T. J., Shepherd, M. C., and Readhead, A. C. S., Astrophys. J. (2001), in press, astro-ph/0104489.

48. Pryke, C., Halverson, N. W., Leitch, E. M., Kovac, J., Carlstrom, J. E., Holzapfel, W. L., and Dragovan, M., Astrophys. J. (2001), in press, astro-ph/0104490.

49. Novak, G., Chuss, D. T., Renbarger, T., Peterson, J. B., Griffin, G. S., Newcomb, M. G., Loewenstein, R. F., Pernic, D., and Dotson, J. L., Astrophys. J. Lett. (2001), submitted, a stro-ph/0109074.

50. LaRosa, T. N., Kassim, N. E., Lazio, T. J. W., and Hyman, S. D., Astron. J. , 119 (2000).

51. Pierce-Price, D., Richer, J. S., Greaves, J. S., Holland, W. S., Jenness, T., Lasenby, A. N., White, G. J., Matthews, H. E., Ward-Thompson, D., Dent, W. R. F., Zylka, R., Mezger, P., Hasegawa, T., Oka, T., Omont, A., and Gilmore, G., Astrophys. J. Lett. , 545 (2000).

52. Kim, S., Martin, C. L., Stark, A. A., and Lane, A. P., American Astronomical Society Meeting 197, BAAS, 32, 4.04 (2000). 
53. Uchida, Y., Shibata, K., and Sofue, Y., Nature, 317, 699 (1985).

54. Kooi, J. W., Man, C., Phillips, T. G., Bumble, B., and LeDuc, H. G., IEEE trans. Microwaves Theory and Techniques, 40, 812 (1992).

55. Engargiola, G., Zmuidzinas, J., and Lo, K.-Y., Rev. Sci. Instr., 65, 1833 (1994).

56. Zmuidzinas, J., and LeDuc, H. G., IEEE Trans. Microwave Theory Tech., 40, 1797 (1992).

57. Walker, C. K., Kooi, J. W., Chan, W., LeDuc, H. G., Schaffer, P. L., Carlstrom, J. E., and Phillips, T. G., Int. J. Infrared and Millimeter Waves, 13, 785 (1992).

58. Kooi, J. W., Chan, M. S., Bumble, B., LeDuc, H. G., Schaffer, P. L., and Phillips, T. G., Int. J. IR and MM Waves, 16 (1995).

59. Honingh, C. E., Hass, S., Hottgenroth, K., Jacobs, J., and Stutzki, J., IEEE Trans. Appl. Superconductivity, 7, 2582 (1997).

60. Walker, C., Groppi, C., Hungerford, A., Kulesa, K., C. an d Jacobs, Graf, U., Schieder, R., Martin, C., and Kooi, J., "PoleSTAR: A 4-channel 810 GHz Array Receiver for AST/RO", in Twelfth Intern. Symp. Space THz Technology, Twelfth Intern. Symp. Space THz Technology, NASA JPL, 2001, in press.

61. Groppi, C., Walker, C., Hungerford, A., Kulesa, C., Jacobs, K., and Kooi, J., "PoleSTAR: An 810 GHz Array Receiver for AST/RO", in Imaging at Radio Through Submillimeter Wavelengths, edited by J. G. Mangum and S. J. E. Radford, ASP Conference Series, San Francisco, 2000, vol. 217, p. 48.

62. Schieder, R., Tolls, V., and Winnewisser, G., Exp. Astron., 1, 101 (1989).

63. Gerecht, E., Musante, C. F., Zhuang, Y., Yngvesson, K. S., Goyette, T., Dickinson, J., Waldman, J., Yagoubov, P. A., Gol'tsman, G. N., Voronov, B. M., and Gershenzon, E. M., IEEE Trans., MTT-47, 2519 (1999).

64. Yngvesson, K. S., Musante, C. F., Ji, M., Rodriguez, F., Zhuang, Y., Gerecht, E., Coulombe, M., Dickinson, J., Goyette, T., Waldman, J., Walker, C. K., Stark, A. A., and Lane, A. P., "Terahertz Receiver with NbN HEB Device (TREND) - A Low-Noise Receiver User Instrument for AST/RO at the South Pole”, Twelfth Intern. Symp. Space THz Technology, 2001, in press.

65. Swain, M. R., Bradford, C. M., Stacey, G. J., Bolatto, A. D., Jackson, J. M., Savage, M., and Davidson, J. A., SPIE, 3354, 480 (1998).

66. NRC, Astronomy and Astrophysics in the New Millennium, National Research Council, National Academy Press, 2001.

67. Stark, A. A., "Design Considerations for Large Detector Arrays on Submillimeter-wave Telescopes", in Radio Telescopes, edited by H. R. Butcher, 2000, vol. 4015 of Proceedings of SPIE, p. 434. 